\title{
Surprise: Unexpected Action Execution and Unexpected Inhibition Recruit the Same Fronto-Basal-Ganglia Network
}

\author{
Alexandra Sebastian, ${ }^{1}$ Anne Maria Konken, ${ }^{1}$ Michael Schaum, ${ }^{2,3}$ Klaus Lieb, ${ }^{1,3}$ Oliver Tüscher, ${ }^{1,3 *}$ and \\ Patrick Jung ${ }^{1,4 *}$ \\ ${ }^{1}$ Department of Psychiatry and Psychotherapy, Focus Program Translational Neuroscience (FTN), University Medical Center of the Johannes \\ Gutenberg University Mainz, 55131 Mainz, Germany, ${ }^{2}$ Brain Imaging Center, MEG Unit, Goethe University Frankfurt am Main, 60528 Frankfurt \\ am Main, Germany, ${ }^{3}$ Leibniz Institute for Resilience Research, 55122 Mainz, Germany, and ${ }^{4}$ Center of Neuropsychiatry Frankfurt-Sachsenhausen, \\ 60594 Frankfurt am Main Germany
}

Unexpected and thus surprising events are omnipresent and oftentimes require adaptive behavior such as unexpected inhibition or unexpected action. The current theory of unexpected events suggests that such unexpected events just like global stopping recruit a fronto-basal-ganglia network. A global suppressive effect impacting ongoing motor responses and cognition is specifically attributed to the subthalamic nucleus (STN). Previous studies either used separate tasks or presented unexpected, task-unrelated stimuli during response inhibition tasks to relate the neural signature of unexpected events to that of stopping. Here, we aimed to test these predictions using a within task design with identical stimulus material for both unexpected action and unexpected inhibition using functional magnetic resonance imaging (fMRI) for the first time. To this end, 32 healthy human participants of both sexes performed a cue-informed go/nogo task comprising expected and unexpected action and inhibition trials during fMRI. Using conjunction, contrast, and Bayesian analyses, we demonstrate that unexpected action elicited by an unexpected go signal and unexpected inhibition elicited by an unexpected nogo signal recruited the same fronto-basal-ganglia network which is usually assigned to stopping. Furthermore, the stronger the unexpected action-related activity in the STN region was the more detrimental was the effect on response times. The present results thus complement earlier findings and provide direct evidence for the unified theory of unexpected events while ruling out alternative task and novelty effects.

Key words: go/nogo task; inferior frontal cortex; response inhibition; subthalamic nucleus; theory of unexpected events

Significance Statement

This is the first study using functional magnetic resonance imaging (fMRI) to test whether unexpected events regardless of whether they require unexpected action or inhibition recruit a fronto-basal-ganglia network just like stopping. In contrast to previous studies, we used identical stimulus material for both conditions within one task. This enabled us to directly test predictions of the current theory of unexpected events and, moreover, to test for condition-specific neural signatures. The present results underpin that both processes recruit the same neural network while excluding alternative task and novelty effects. The simple task design thus provides an avenue to studying surprise as a pure form of reactive inhibition in neuropsychiatric patients displaying inhibitory deficits who often have a limited testing capacity.

Received July 1, 2020; revised 0ct. 21, 2020; accepted Nov. 20, 2020.

Author contributions: A.S., K.L., 0.T., and P.J. designed research; A.S. and A.M.K. performed research; A.S., A.M.K., and M.S. analyzed data; A.S. wrote the paper.

This work was supported by internal grants of the MAIFOR program and the research Focus Translational Neurosciences (FTN) of the Johannes Gutenberg University Medical Center Mainz, Germany (P.J.) and the German Research Foundation (Deutsche Forschungsgemeinschaft, DFG) Grant CRC 1193 C04 (to 0.T.). Findings presented in this study are partly included in the doctoral thesis of A.M.K.

*0.T. and P.J. contributed equally to this work.

The authors declare no competing financial interests.

Correspondence should be addressed to Alexandra Sebastian at alexandra.sebastian@unimedizin-mainz.de.

https://doi.org/10.1523/JNEUROSCI.1681-20.2020

Copyright $\odot 2021$ the authors

\section{Introduction}

Unexpected events are omnipresent and usually require rapid adaptation of ongoing behavior. For example, a car driver must brake when a kid runs onto the road. Such situations require reactive control when unexpected stimuli signal the need to rapidly cancel ongoing actions and to initiate new, situationally more appropriate behaviors. Reactive control is thus necessary to avoid harmful consequences, making it essential for adaptive behavior.

The neural underpinnings of reactive motor control have been extensively investigated using response inhibition tasks, such as stop-signal (Logan and Cowan, 1984) and go/nogo tasks (Donders, 1969). In these tasks, prepotent or ongoing actions 
must be inhibited in response to infrequent signals (Aron, 2011; Bari and Robbins, 2013). The onset of the stop-signal differs between the two tasks: while the nogo-signal is presented instead of or simultaneously with the go-signal in the go/nogo task, the stop-signal in the stop-signal task is presented at some delay after the go signal. The two tasks thus capture different subcomponents of response inhibition, i.e., action restraint and action cancellation, respectively (Schachar et al., 2007). Nevertheless, neuroimaging studies yielded converging evidence of overlapping neural networks during response inhibition tasks (Levy and Wagner, 2011; Swick et al., 2011; Sebastian et al., 2013; Cieslik et al., 2015). This shared stopping network comprises the right inferior frontal gyrus (IFG) and anterior insula, the presupplementary motor area (preSMA), and basal ganglia [subthalamic nucleus (STN) and striatum; Duann et al., 2009; Aron, 2011; Levy and Wagner, 2011; Aron et al., 2014; Sebastian et al., 2016]. STN involvement has mainly been discussed in action cancellation, but has also been demonstrated to be implicated in action restraint (Ballanger et al., 2009; Georgiev et al., 2016; Marmor et al., 2020).

Wessel and Aron (2017) recently suggested a unified, neuroanatomically grounded theory of unexpected events. They propose that the neural stopping network [i.e., right inferior frontal cortex (IFC), pre-SMA, and STN] or a closely analogous neural network is recruited by unexpected events. More specifically, unexpected events interrupt action and cognition by this global suppressive network with downstream effects on basal-ganglia output resulting in motor slowing and cognitive distraction. Unexpected events may trigger purely reactive inhibition as no proactive stopping tendencies are involved (Wessel, 2018). The theory builds on a related theory by Corbetta and Shulman (2002), who proposed that a right-lateralized circuit breaker including the right IFC interrupts ongoing cognition after unexpected events allowing for reorienting attention to behaviorally relevant stimuli. The unified theory of unexpected events goes beyond these suggestions in three respects. First, Wessel and Aron (2017) suggest that in the surprise-interruption-reorienting sequence the interruption part recruits pre-SMA and STN in addition to right IFC. Second, they suggest that the interrupt system and attentional reorienting are independent. Third, global interruption by the stopping network should temporally precede the attentional reorienting after unexpected events. In the present study we aimed to test the first of these notions based on the example of unexpected inhibition and unexpected action execution. Unexpected action refers to actions which are not anticipated and planned but suddenly required. In the above-mentioned example, chatting adults might be standing at the roadside. When the approaching car driver honks the horn (= unexpected event/surprise) to warn the child running into the road, they stop chatting (= interruption), direct their attention to the situation on the road (= reorientation), and start running after the child (= unexpected action). As outlined at the beginning, the braking of the driver when he notices the child is an example of unexpected inhibition. Unexpected action and unexpected inhibition hence result from the surprise-interruption-reorienting sequence proposed by Wessel and Aron (2017).

Previous studies either used separate tasks or presented taskunrelated surprising stimuli during response inhibition tasks to test for a common neural signature of response inhibition and unexpected events (Wessel and Aron, 2013; Wessel et al., 2016; Dutra et al., 2018; Wagner et al., 2018; Wessel, 2018; Wessel and
Huber, 2019). Such an approach is particularly suited to assess whether a putatively suppressive component associated with stopping is also recruited by surprise. However, it is not suitable for investigating commonalities and differences in the respective neural networks (cf. also Wessel et al., 2016). In this first functional magnetic resonance imaging (fMRI) study addressing the theory of unexpected events, we thus chose a within task design with identical stimulus material. This enabled us to test the hypothesis that a common neural network usually assigned to response inhibition is involved in interrupting ongoing processing on unexpected events regardless of whether the unexpected event requires executing (unexpected action) or withholding (unexpected inhibition) a response. More importantly, the cued go/nogo task design allowed us to study both, the commonalities and the differences in the neural architecture of unexpected inhibition and unexpected action while excluding task and novelty effects, thus complementing previous findings. In line with the unified theory of unexpected events, we hypothesized that unexpected action and unexpected inhibition would equally recruit the fronto-basal-ganglia stopping network as indexed by conjunction and contrast analyses. We further expected a positive correlation of inhibition-related STN activity and response slowing following unexpected events.

\section{Materials and Methods \\ Participants}

In total, 37 healthy participants participated in the study. Two participants had to be excluded because of excessive head movement inside the scanner, one because of technical problems and another two participants were excluded because of incidentally discovered brain abnormalities. The remaining 32 participants ( 16 female) had a mean age of 24.97 years $(\mathrm{SD}=1.40$, range: $22-28$ years). All individual participants included in the study were screened for factors contradicting MRI scanning and provided written informed consent before participation. They were all right-handed as determined by the Edinburgh Handedness Inventory (Oldfield, 1971), had normal or corrected to normal vision, and were free of psychotropic medication. None of the participants had a history or current evidence of psychiatric or neurologic diseases. The study was approved by the local ethics committee and participants were financially compensated for their time.

\section{Experimental design}

We employed a cued go/nogo paradigm (Fig. 1) using Presentation software (version 18.0; www.neurobs.com). Before the scanning session, participants received a brief training session to make sure that the participants correctly understood the task instructions and to familiarize them with the task before the scanning session. All participants accomplished four runs of the task during the scanning session.

Throughout scanning, participants were asked to hold an MR compatible response button box in their right hand and to respond to the stimuli by pressing a response button with the right index finger. Before the beginning of each run, instructions were given orally. Instructions equally stressed speed and accuracy of responding.

The task comprised four conditions (Fig. 1): expected go (go cue go target, $37.5 \%$ ), expected nogo (nogo cue - nogo target, 37.5\%), unexpected go (nogo cue - go target, $12.5 \%$ ), and unexpected nogo (go cue nogo target, $12.5 \%$ ). One run consisted of 80 trials so that in total 120 expected go trials, 120 expected nogo trials, 40 unexpected go trials, and 40 unexpected nogo trials were presented. At the beginning of each trial, a white fixation cross was presented on gray background in the center of the screen for a randomly varied duration of $2500-3500 \mathrm{~ms}$. Then, a filled circle was presented for a randomly varied duration of 800 $1200 \mathrm{~ms}$ that was either a predictive go cue (yellow circle) or a predictive nogo cue (orange circle). A go cue was followed by a go target (filled blue circle) with a probability of $75 \%$ (expected go trial) whereas a nogo target (filled green circle) was presented with a probability of $25 \%$ 


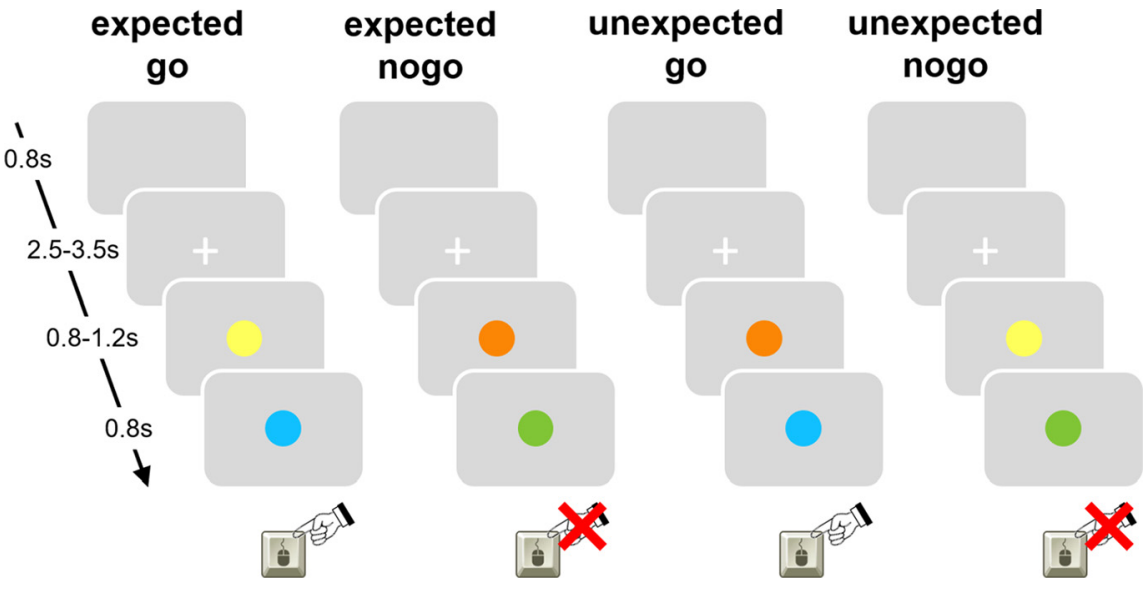

Figure 1. Cued go/nogo task: go cues in yellow, nogo cues in orange, go targets in blue, nogo targets in green. The attribution of color to trial type was counterbalanced across participants. On unexpected nogo trials, subsequently after an invalid go cue a nogo target was displayed instructing participants to withhold the response (12.5\%). On unexpected go trials, subsequently after an invalid nogo cue a go target was displayed instructing participants to perform the response (12.5\%). The remaining trials were expected nogo trials $(37.5 \%)$ and expected go trials $(37.5 \%)$ in which the cue validly predicted the target stimulus.

(unexpected nogo trial). Likewise, a nogo cue was followed by nogo target with a probability of $75 \%$ (expected nogo trial) whereas a go target was presented with a probability of $25 \%$ (unexpected go trial). The target stimulus was displayed for a maximum of $800 \mathrm{~ms}$ [equivalent to the maximum permitted reaction time (RT)] or until a button press was performed. In case of a button press, the target stimulus vanished and the screen remained blank until the end of the trial. At the end of each trial, a blank screen was presented for $800 \mathrm{~ms}$. Participants were instructed that cue stimuli were highly predictive of target stimuli. They were asked to use the information of the cue stimulus to best possibly prepare the response to the target stimulus and to press the button in case of a go target only and to withhold a response in case of a nogo target. The attribution of color to trial type was counterbalanced across participants.

\section{Statistical analyses}

Behavioral data analyses

Behavioral data (RT and accuracy) were collected by the Presentation software, and analyzed using MATLAB 2013b (The MathWorks Inc.), SPSS software (IBM SPSS version 23), and JASP (version 0.11.1.0; JASP team, 2019; https://jasp-stats.org). Measures of interest were mean RT in correct expected and unexpected go trials and percentage of errors (i.e., omission errors on expected and unexpected go trials and commission errors on expected and unexpected nogo trials). The effect of unexpectedness on response time (surprise effect) was computed by subtracting expected go RT from unexpected go RT. Responses given after the cue but before the target were classified as pretarget responses. In case of non-sphericity, corrected df and $p$ values are reported (GreenhouseGeisser $\varepsilon$ correction).

\section{MRI data acquisition}

Images were acquired on a Magnetom Trio syngo $3 \mathrm{~T}$ system (Siemens) equipped with 32-channel head coil for signal reception. Stimuli were projected on a screen at the head end of the scanner bore and were viewed with the aid of a mirror mounted on the head coil. Foam padding was used to limit head motion within the coil. A high-resolution T1-weighted anatomic data set was obtained using a 3D magnetization prepared rapid acquisition gradient echo (MPRAGE) sequence for registration purposes $\left(\mathrm{TR}=1900 \mathrm{~ms}, \mathrm{TE}=2.52 \mathrm{~ms}\right.$, flip angle $=9^{\circ}, \mathrm{FOV}=256 \mathrm{~mm}, 176$ sagittal slices, voxel size $1 \times 1 \times 1 \mathrm{~mm}^{3}$ ). Functional MRI images were obtained using $\mathrm{T} 2 *$-weighted echoplanar imaging (EPI) sequence $\left(\mathrm{TR}=2500 \mathrm{~ms}, \mathrm{TE}=30 \mathrm{~ms}\right.$, flip angle $=90^{\circ}, \mathrm{FOV}=192$ $\mathrm{mm}, 38$ slices, voxel size $=3 \times 3 \times 3 \mathrm{~mm}^{3}$ ).
Preprocessing of fMRI data

SPM12 (www.fil.ion.ucl.ac.uk/spm/software/ spm12/) was used to conduct all image preprocessing and statistical analyses, running with MATLAB 2013b (The MathWorks Inc.). Images were screened for motion artifacts before data analysis. Excessive head motion was observed in two of the participants who were consequently excluded from all data analyses. Next, images were manually reoriented to the T1 template of SPM. The first five functional images of each run were discarded to allow for equilibrium effects. Then, several preprocessing steps were conducted on the remaining functional images. First, images were realigned to the first image of the first run, using a 6-df rigid body transformation. The realigned functional images were coregistered to the individual anatomic T1 image using affine transformations. Subsequently, the anatomic image was spatially normalized (linear and nonlinear transformations) into the reference system of the Montreal Neurologic Institute (MNI)'s reference brain using standard templates, and normalization parameters were applied to all functional images. Finally, the normalized functional data were smoothed with a three-dimensional isotropic Gaussian kernel (8-mm full width at half maximum) to enhance signal-to-noise ratio and to allow for residual differences in functional neuroanatomy between subjects.

\section{Single subject analysis}

A linear regression model (general linear model; GLM) was fitted to the fMRI data of each subject. All events were modeled as stick functions at stimulus onset of the target stimulus and convolved with a canonical hemodynamic response function. The model included a high-pass filter with a cutoff period of $128 \mathrm{~s}$ to remove drifts or other low frequency artifacts in the time series. After convolution with a canonical hemodynamic response function, four event types were modeled as regressors of interest: correct expected go ("go cue - go target"), correct unexpected go ("nogo cue - go target"), correct expected nogo ("nogo cue - nogo target"), correct unexpected nogo ("go cue - nogo target"). Incorrect reactions (i.e., button press following a nogo target, no button press following a go target, pretarget responses) for each condition were modeled as regressors of no interest. In addition, the six covariates containing the realignment parameters capturing the participants' movements during the experiment were included in the model.

\section{Group analysis}

To identify neural underpinnings of unexpected inhibition (inhibition elicited by unexpected nogo-stimuli) and unexpected action (action execution elicited by unexpected go-stimuli), we subjected the four event types, that had been modeled on first level (expected go: "go cue - go target"; unexpected go: "nogo cue - go target"; expected nogo: "nogo cue - nogo target"; unexpected nogo: "go cue - nogo target") to a fullfactorial model. We assessed neural activation patterns underlying unexpected inhibition using the contrast "unexpected nogo $>$ expected nogo." This contrast is particularly suited to identify the neural architecture of unexpectedness in the inhibitory domain. While both experimental conditions require inhibition, they differ regarding the degree of unexpectedness. Since no button press is required in neither of the trial types, resulting brain activity is not contaminated by a motor response. Similarly, we assessed neural underpinnings of unexpected action using the contrast "unexpected go $>$ expected go." Both conditions require action execution but differ regarding the degree of unexpectedness.

In order to identify regions mutually activated during unexpected inhibition and unexpected action, we conducted a logical AND conjunction analysis ("conjunction null"; Nichols et al., 2005) of the respective 
contrasts (i.e., "unexpected nogo > expected nogo" $\cap$ "unexpected go > expected go").

To test for differences in activation patterns associated with unexpected inhibition and unexpected action the contrasts "unexpected nogo $>$ unexpected go" and "unexpected go $>$ unexpected nogo" were used, respectively. In addition, the contrasts capturing unexpected inhibition ("unexpected nogo $>$ expected nogo") and unexpected action ("unexpected go > expected go") were computed on first level and contrasted on second level using a paired $t$ test in order control for expectancyrelated and motor-related processes.

Significant effects for each condition were assessed using $t$ statistics. The respective group results were thresholded at $p<0.05$ corrected for multiple comparisons [family wise error (FWE), correction at peak level] and $\mathrm{k}>5$ contiguous voxels. The SPM anatomy toolbox 2.0 (Eickhoff et al., 2005, 2006, 2007) was used to allocate significant clusters of activation to predefined anatomic regions. Small volume corrections (SVCs) were performed for two subcortical key regions of the neural response inhibition network (Aron, 2011), namely the right STN region and the right striatum, using binarized masks from the probabilistic atlas from Keuken et al. (2014). The probabilistic masks were thresholded at $10 \%$. We limited the SVC to right hemispheric regions of interest (ROIs) for two reasons. First, the neural stopping network is right lateralized (Cai et al., 2014). Second, participants responded with the right hand which should mainly be reflected in brain activity in the contralateral hemisphere (Marsden et al., 1985; Lanska, 2009). We reasoned that signal in left-hemispheric basal ganglia ROIs might thus be more strongly intermixed with neural underpinnings of motor responses in the respective conditions. We used an initial cluster forming threshold of $p_{\text {uncorrected }}<$ 0.05 and then applied the SVCs. Small volume corrected activations were regarded as significant if they survived $p<0.05$ for an FWE correction after correcting for multiple comparisons (i.e., two ROIs) using a Bonferroni correction. Notably, BOLD signal may not unequivocally be localized to the STN using 3 T fMRI because of its small size and proximity to other small structures such as the substantia nigra (de Hollander et al., 2015). However, given the prominent role of the STN in the theory of unexpected events (Wessel and Aron, 2017), we included the STN as a ROI to provide preliminary insights. Importantly, the anatomic masks were derived from ultra-high resolution in vivo anatomic imaging using 7 T MRI atlas increasing regional specificity, whereas previous studies usually used a box exceeding the STN volume many times (de Hollander et al., 2015).

Finally, we used Bayesian statistics to provide evidence for (null hypothesis, $\mathrm{H}_{0}$ ) or against (alternative hypothesis, $\mathrm{H}_{1}$ ) the assumption that regions that are usually assigned to the stopping network are equally recruited when in interrupting ongoing processing regardless of whether an unexpected event requires executing (unexpected action execution elicited by unexpected go stimuli) or withholding a response (unexpected inhibition elicited by unexpected nogo stimuli; Gallistel, 2009; Wagenmakers et al., 2016). Therefore, we conducted a ROI analysis within the key regions of the response inhibition network (Aron, 2011). The following masks were used: the right IFG pars opercularis and pars triangularis ROIs from the Harvard-Oxford atlas included in FSL (Desikan et al., 2006); the pre-SMA ROI was provided by Boekel et al. (2017) and was drawn in MNI space by using the coordinates reported by Johansen-Berg et al. (2004); the right STN and right striatum ROIs from the probabilistic atlas from Keuken et al. (2014). All probabilistic masks were thresholded at $10 \%$. Percent signal change during unexpected nogo and unexpected go trials was extracted using rfxplot (Gläscher, 2009). We computed Bayesian paired $t$ tests using JASP version 0.11.1.0 (JASP team, 2019; https://jasp-stats.org). Since we had no prior information, we used the default Cauchy prior provided by JASP (scale $=0.707)$.

\section{Brain-behavior relationship}

In order to test the hypothesis that stronger STN activity evoked by unexpected events increases response slowing as suggested by Wessel and Aron (2017), we computed the Pearson correlation coefficient of percent signal change in the right STN region during unexpected go trials and the effect of unexpectedness on response time (surprise effect:

\section{Table 1. Behavioral results}

\begin{tabular}{lrr}
\hline & \multicolumn{1}{l}{ M } & \multicolumn{1}{c}{ SD } \\
\hline RT expected go (go cue - go target); ms & 271.58 & 33.72 \\
RT unexpected go (nogo cue - go target); ms & 381.76 & 52.06 \\
Surprise effect (ms) & 110.18 & 58.40 \\
Omission errors expected go (go cue - go target); \% & 0.23 & 0.48 \\
Omission errors unexpected go (nogo cue - go target); \% & 1.33 & 2.01 \\
Commission errors expected nogo (nogo cue - nogo target); \% & 0.23 & 0.48 \\
Commission errors unexpected nogo (go cue - nogo target); \% & 28.13 & 24.02 \\
\hline
\end{tabular}

RTs (in milliseconds) and error rates of 32 participants. Surprise effect was calculated by subtracting expected go RT from unexpected go RT. Percentage error is estimated by dividing the number of incorrect/omitted trials by the total number of the respective trial type.

RT difference between unexpected and expected go trials). Based on our hypothesis and previous findings (Wessel et al., 2016), we tested for a positive relationship.

\section{Results}

\section{Behavioral data}

Participants performed accurately as indicated by low error rates on go as well as on nogo trials. RTs and error rates are summarized in Table 1.

Mean correct reactions (mean \pm SD) were $97.99 \pm 3.43 \%$ in the expected go condition, $99.77 \pm 0.49 \%$ in the expected nogo condition, $98.67 \pm 2.01 \%$ in the unexpected go condition, and $70.31 \pm 25.61 \%$ in the unexpected nogo condition. To compare RTs and error rates between conditions we used paired $t$ tests. These revealed better performance in expected as compared with unexpected conditions. Mean RT in unexpected go trials was significantly slower than in expected go trials $\left[t_{(31)}=10.67\right.$, $p<0.001, d_{\text {Cohen }}=1.886,95 \%$ confidence interval $(\mathrm{CI}): 1.299$, 2.463]. Participants made more omission errors in the unexpected go condition than in the expected go condition $\left(t_{(31)}=\right.$ 3.109, $p=0.004$, Cohen's $d=0.550)$. Similarly, participants made more commission errors in the unexpected nogo condition than in the expected nogo condition $\left(t_{(31)}=6.644\right.$, $p<0.001$, Cohen's $d=1.175$ ). Pretarget responses (mean \pm SD) were only present in conditions containing a go cue (expected go condition: $1.77 \pm 3.48 \%$; unexpected nogo condition: $1.56 \pm 4.25 \%)$ and were equally probable in both conditions $\left(t_{(31)}=0.458, p=0.650\right.$, Cohen's $\left.d=0.081\right)$.

\section{Imaging data}

We assessed whether unexpected inhibition ("unexpected nogo $>$ expected nogo") and unexpected action ("unexpected go $>$ expected go") depend on common networks, unique networks, or a combination of both. As shown in Figure 2 and Table 2, unexpected inhibition and unexpected action both elicited prominent brain activity in multiple prefrontal regions, including the IFG/anterior insula, middle frontal gyrus including inferior frontal junction (IFJ), pre-SMA, as well as in inferior parietal regions. For both contrasts significant activation was found in the right STN region and striatum or, more precisely, the caudate nucleus using SVC (Fig. 3). However, after applying Bonferroni correction, activity in right STN and right striatum remained significant during unexpected inhibition only. A conjunction analysis ("unexpected go $>$ expected go" $\cap$ "unexpected nogo $>$ expected nogo") confirmed that the interruption of processing following unexpected events, regardless of whether these required unexpected inhibition or unexpected action execution mutually relied on the network that is 


\section{i) unexpected go > expected go}
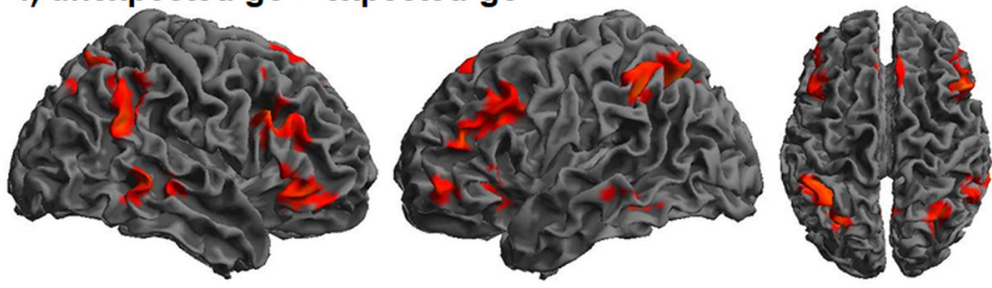

ii) unexpected nogo > expected nogo
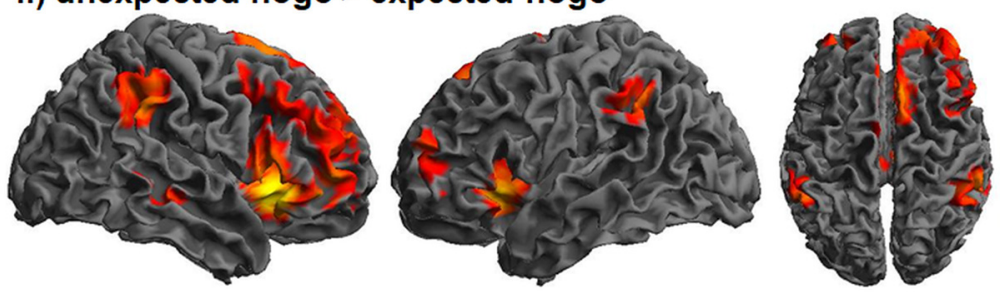

ii) conjunction
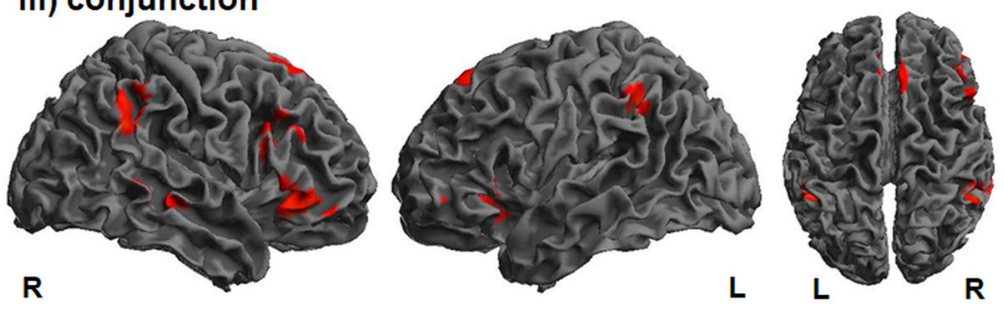

0

14

Figure 2. Activation maps for unexpected action (unexpected go $>$ expected go; top row), unexpected inhibition (unexpected nogo > expected nogo; middle row) and the conjunction of unexpected action and unexpected inhibition with overlapping activity in bilateral ventrolateral prefrontal cortex and pre-SMA. The color bar represents T scores. $p_{F W E}<0.05, \mathrm{k}=5$.

typically assigned to response inhibition (Aron, 2011; Sebastian et al., 2016; Fig. 2; Table 2).

The ROI analysis with Bayesian statistics (Fig. 4) revealed only anecdotal evidence for differences in activation between unexpected nogo and unexpected go trials for the right IFG (pars opercularis) and the right striatum. In contrast, there was moderate evidence in favor of the null hypothesis, i.e., activity in the right IFG (pars triangularis), pre-SMA and right STN is not different between unexpected inhibition and unexpected action. For these ROIs, the data are three to five times more likely under the null hypothesis than under the alternative hypothesis.

Finally, we assessed whether unexpected inhibition and unexpected action relied on different networks. Contrasting unexpected nogo to unexpected go ("go cue - nogo target" > "nogo cue - go target") revealed no significant results at all. Contrasting unexpected go to unexpected nogo ("nogo cue - go target" > "go cue - nogo target") resulted in activity in a motor network including left primary motor cortex, right cerebellum and thalamus (Table 3), but revealed no activity in key regions of the stopping network such as the right ventrolateral IFG, pre-SMA, or STN. Importantly, this contrast did not control for motor-related processes, since a button press was required in the unexpected go condition but not in the unexpected nogo condition. To control for expectancy-related and motor-related processes, we thus computed paired $t$ tests. Neither the paired $t$ test for unexpected action $>$ unexpected inhibition (i.e., "unexpected go - expected go" > "unexpected nogo - expected nogo") nor the inverse contrast for unexpected inhibition $>$ unexpected action (i.e., "unexpected nogo - expected nogo" > "unexpected go - expected go") revealed any significant clusters of activation.

\section{Brain behavior relationship}

In order to test the hypothesis that the stronger the STN recruitment, the stronger is the detrimental effect on behavior, we correlated percent signal change in the right STN region in the unexpected go condition with the effect of unexpectedness on response time (surprise effect, i.e., the difference between RT in unexpected and expected go conditions). Percent signal change in right STN region moderately correlated with the surprise effect ( $r=0.304, p=0.046,95 \%$ CI: 0.008 , $1.000 ;$ Fig. 5). This suggests that the more strongly STN is recruited, the more strongly the interruption elicited by unexpected events affects action and cognition which is reflected in stronger response slowing on unexpected events.

Given the width of the CI and the fact that brain-behavior correlations in small samples may be sensitive to outliers which can bias the estimates (Cremers et al., 2017), we performed an outlier analysis on the percent signal change in the right STN region during unexpected go trials. Using the interquartile range (IQR)-based method and the standard deviation-based method, we observed no extreme outliers [no values outside the range $\mathrm{Q} 1-(3 * \mathrm{IQR})$ - Q3+ $(3 * I Q R)$ and no value exceeding $\mathrm{SD}>|3|]$. The values of two participants, however, were identified as mild outliers [values larger than Q3+ (1.5*IQR) and SD >2.58]. After excluding the values of these participants, the brain behavior correlation was no longer significant $(r=0.155, p=0.206,95 \%$ CI: $-0.159,1.000)$.

\section{Discussion}

We directly tested the hypothesis of a global neural network that is recruited by a variety of unexpected events (Wessel and Aron, 2017). Unexpected inhibition and unexpected action both recruited a common fronto-basal-ganglia network typically assigned to stopping (Aron and Poldrack, 2006; Levy and Wagner, 2011; Sebastian et al., 2013; Jahanshahi et al., 2015; Fig. 4). This network comprised cortical activity in the right IFG/anterior insula and pre-SMA (Fig. 2) and subcortical activity in the right STN region and caudate nucleus (Fig. 3). Contrasting unexpected nogo and unexpected go conditions revealed no differences in activation patterns when controlling for a motor response. Moreover, the stronger the activity in the STN region was on unexpected go trials, the more detrimental the behavior was affected as reflected in stronger response slowing (Fig. 5). The present findings thus provide direct evidence for the predictions of the theory of unexpected events (Wessel and Aron, 2017) and complement and replicate previous indirect evidence (Wessel and Aron, 2013; Wessel et al., 2016; Dutra et al., 2018; Wagner et al., 2018).

Wessel and Aron (2017) proposed that unexpected events recruit the fronto-basal-ganglia stopping network. They suggest that this results in a global suppressive effect that rapidly interrupts current behavior and cognition, thus allowing attentional reorienting. The global suppressive effect is thought to result 
Table 2. Brain activation during unexpected action and unexpected inhibition

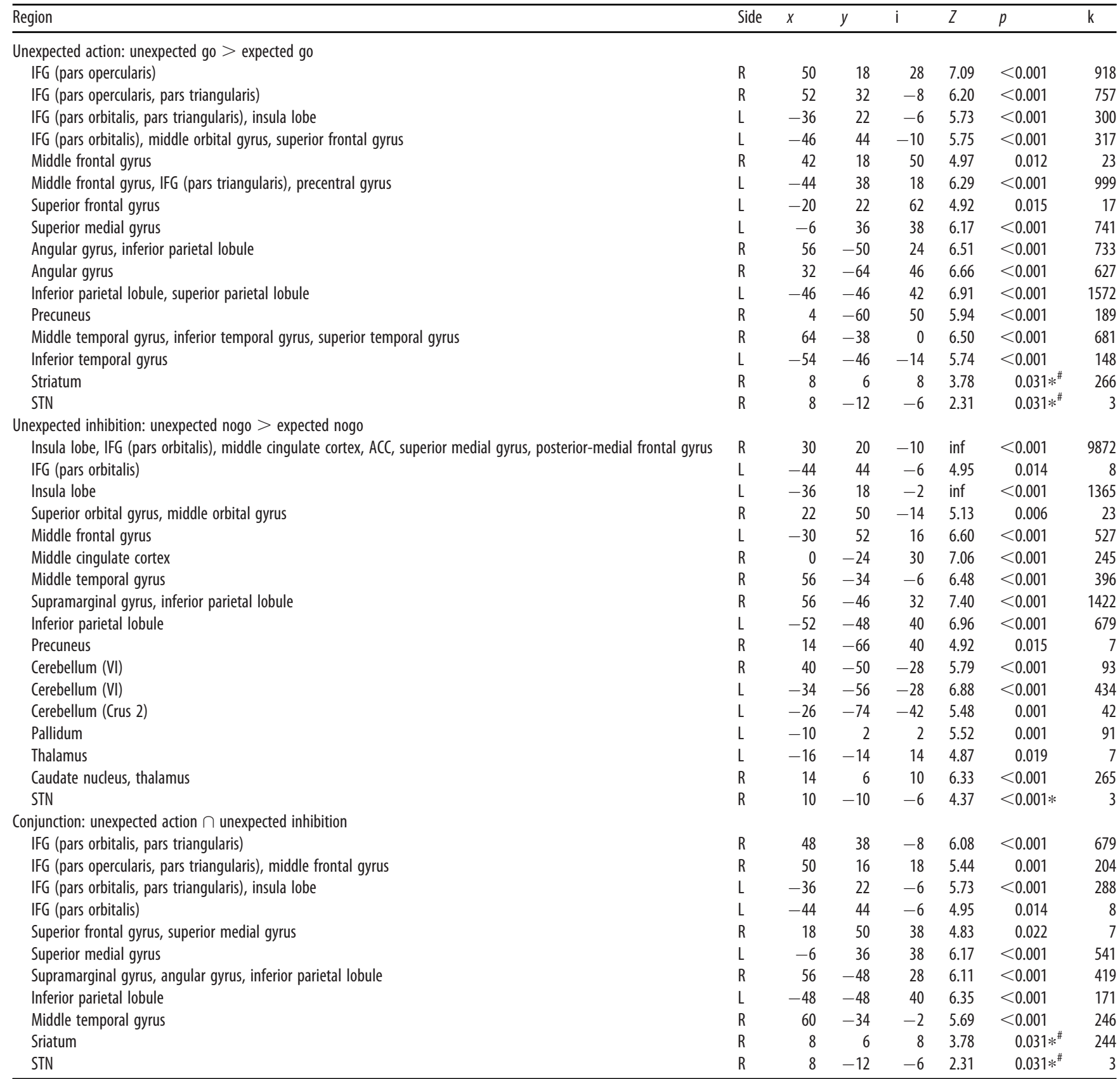

Local maxima of brain activations during unexpected action ("nogo cue - go target" > "go cue - go target"), unexpected inhibition ("go cue - nogo target" > "nogo cue - nogo target") and the conjunction of both contrasts in MNI $x-, y$-, and $z$-coordinates with associated Z-score $\left(p_{F W E}<0.05, k>5\right)$ and cluster extent in number of voxel (k). R, right; L, left; * small volume corrected; \# not significant after Bonferroni correction for multiple comparisons.

from prefrontal projections to the STN with a putative global suppressive effect on basal-ganglia output, hence suppressing thalamocortical drive to the motor system. In line with these predictions, unexpected action and unexpected inhibition following unexpected events were presently associated with activity in prefrontal regions including right IFG and pre-SMA (Fig. 2) as well as in striatum and STN region (Fig. 3). Moreover, Bayesian statistics reveal that activity in these regions was comparable in both conditions (Fig. 4). Behavioral data also support this notion as unexpected go responses were delayed in comparison to expected go responses. Moreover, this response slowing correlated with STN activity which provides further support for the assumption of a global suppressive basal ganglia effect and its behavioral relevance.
A particular strength of the present study is that unexpected action and unexpected inhibition were probed within one experimental paradigm using identical stimulus material. Previous studies either used separate tasks to assess response inhibition and surprise and then tested for a common neural signature, or used a hybrid task design with different stimulus material mainly by means of electroencephalography and independent component analysis (Wessel and Aron, 2013; Wessel et al., 2016; Dutra et al., 2018; Wessel and Huber, 2019). Despite some advantages this approach cannot be used to investigate all commonalities and differences of the respective neural underpinnings (cf. also Wessel et al., 2016). One advantage of electrophysiological methods is the high temporal resolution, which comes, however, at the expense of spatial resolution. The present fMRI study thus 

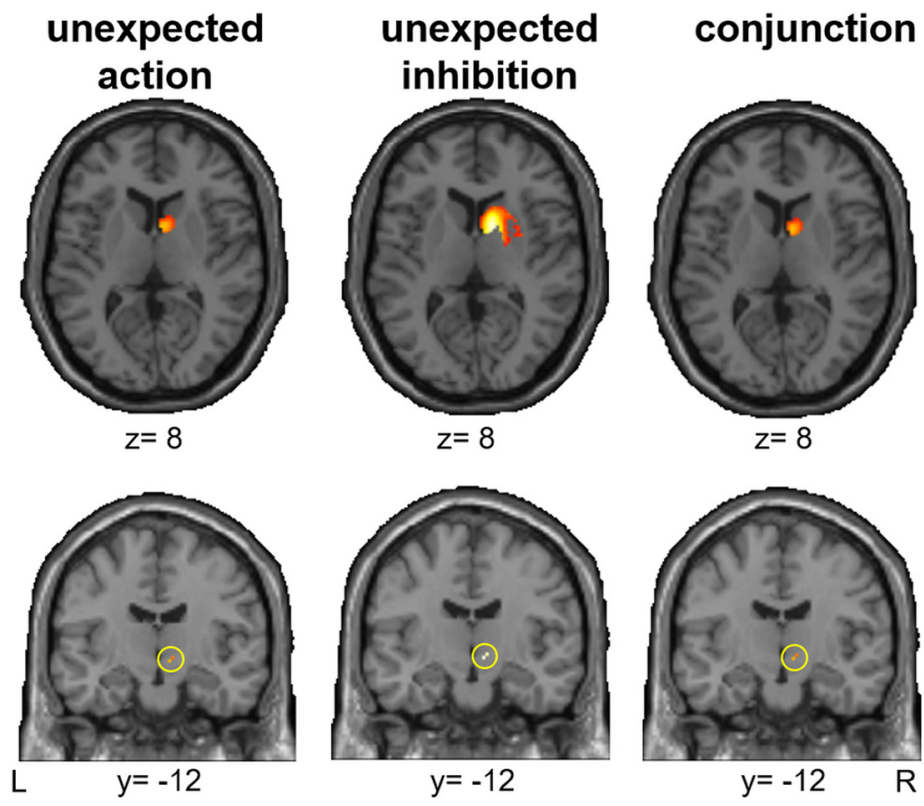

Figure 3. Significant activation ( $p_{\text {FWE }}$ small volume corrected) in the right striatum (top panel) and right STN region (bottom panel) for unexpected action (unexpected go $>$ expected go; left), unexpected inhibition (unexpected nogo > expected nogo; middle) and the conjunction of unexpected action and unexpected inhibition (right). After Bonferroni correction for multiple comparisons, only right striatum and right STN during unexpected inhibition remained significant. The color bar represents $T$ scores.

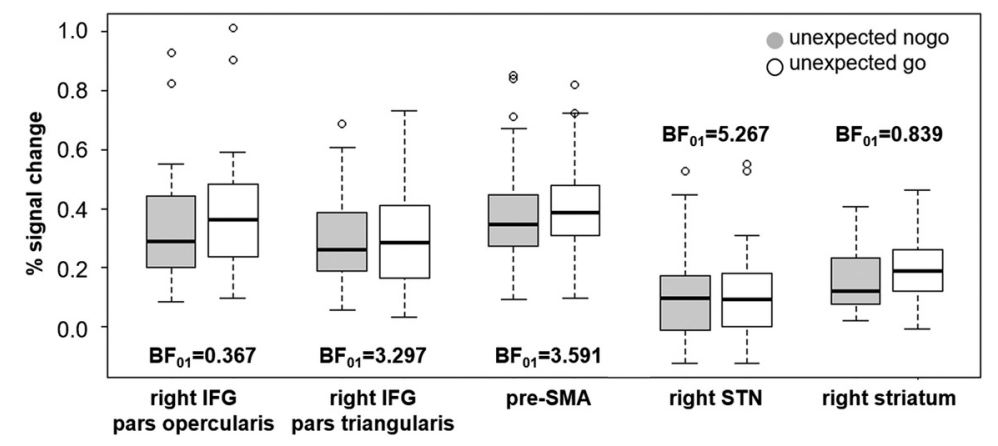

Figure 4. Percent signal change for unexpected nogo (light gray) and unexpected go (white) conditions. Bayes factors $\left(\mathrm{BF}_{01}\right)$ resulting from paired $t$ tests suggest moderate evidence for the null hypothesis (i.e., $\%$ signal change is not different for both conditions) for right IFG pars triangularis, pre-SMA, and right STN, whereas evidence for right IFG pars opercularis and right striatum is inconclusive.

complements and extends previous findings. Using identical stimulus material enabled us to not only test for a common neural signature of unexpected action execution and inhibition elicited by unexpected events. Moreover, we could directly assess commonalities and differences of the respective networks while ruling out stimulus or task effects. Notably, apart from motor effects when not controlling for a motor response, we observed no differences in brain activation patterns for unexpected action and unexpected inhibition. In addition, using Bayesian statistics we provide evidence that unexpected action and unexpected inhibition equally recruit key nodes of the stopping network, namely right IFG, pre-SMA, and STN. The present task design furthermore excludes that findings related to unexpectedness may alternatively be ascribed to novelty processing. Although unexpectedness and novelty often co-occur, they can be clearly distinguished: unexpectedness refers to a violation of prediction, whereas novelty refers to the quality of not being previously encountered (Barto et al., 2013). This distinction is important,
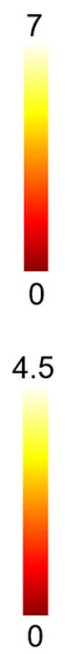

since novelty processing has been associated with a similar fronto-basal-ganglia network including lateral prefrontal regions and striatum (Ranganath and Rainer, 2003; Geiger et al., 2018). Here, go targets were identical regardless of whether they were displayed as predicted by go cues or unexpectedly after nogo cues. The same principle applies to nogo targets. In addition, allocation of color to experimental conditions was counterbalanced across participants. Thus, present findings are unequivocally related to unexpectedness and not to novelty.

Another line of research has assessed the neural signature of surprise in the context of reinforcement learning (Chumbley et al., 2014; Ferdinand and Opitz, 2014; Fouragnan et al., 2017). Converging evidence suggests that prediction error and associated learning is driven by two distinct components (i.e., its valence and its surprise) and that these components are reflected in separate neural signatures (Fouragnan et al., 2018). More specifically, a recent meta-analysis linked surprise to a network comprising, inter alia, the right anterior insula and right IFC, midcingulate cortex/preSMA, dorsal striatum, inferior parietal lobule and middle temporal gyrus (Fouragnan et al., 2018) and thus to a network largely overlapping with the network resulting from our conjunction analysis. Our present finding that both, unexpected action and unexpected inhibition, elicit striatal activity together with the abovementioned evidence suggests that unexpected, surprising events may not only recruit STN but also caudate nucleus. The concept of surprise in the context of reinforcement learning and within the theory of unexpected events are not mutually exclusive specifically with respect to unexpected action outcomes. It is thus not surprising that the respective neural signatures largely overlap indicating neurobiological similarity. Nevertheless, subtle differences exist: the surprise component associated with reinforcement learning relates to the extent of the strength of association between expectations and outcome (den Ouden et al., 2012; Niv et al., 2015; Collins and Frank, 2016; as reviewed by Fouragnan et al., 2018). Wessel and Aron (2017), however, focus on adjustments in the behavioral and cognitive domain regardless of the strength of association. One study assessed Bayesian surprise specifically in the context of stopping and linked it to dorsal anterior cingulate cortex (ACC) activity (Ide et al., 2013). Interestingly, only unexpected inhibition but not unexpected action elicited ACC activity in the present study, suggesting that dorsal ACC might specifically be linked to surprise in the context response inhibition. In contrast to that notion, ACC has also been associated with surprise-encoding in reinforcement learning (Fouragnan et al., 2018). Future meta-analytic approaches are necessary for a more comprehensive understanding of the neural architecture of surprise in different contexts.

The present findings call for a more detailed discussion of the (in-)dependence of interruption and attentional reorienting processes within the cascade following unexpected events. In 
Table 3. Brain activation during unexpected go $>$ unexpected nogo

\begin{tabular}{|c|c|c|c|c|c|c|c|}
\hline Region & Side & $x$ & $y$ & $z$ & $Z$ & $p$ & k \\
\hline IFG (pars opercularis) & $\mathrm{R}$ & 60 & 12 & 24 & 4.83 & 0.023 & 12 \\
\hline Rolandic operculum, insula lobe & $\mathrm{R}$ & 40 & -4 & 14 & 6.27 & $<0.001$ & 225 \\
\hline Rolandic operculum, postcentral gyrus, Insula lobe & $\mathrm{L}$ & -40 & -4 & 16 & 7.58 & $<0.001$ & 1261 \\
\hline Precentral gyrus & L & -58 & 8 & 23 & 5.68 & $<0.001$ & 85 \\
\hline Postcentral gyrus & $\mathrm{R}$ & 56 & -12 & 20 & 4.93 & 0.014 & 20 \\
\hline Postcentral gyrus & $\mathrm{R}$ & 56 & -14 & 44 & 4.75 & 0.031 & 7 \\
\hline Cerebellum (IV-V), cerebellar vermis (4/5) & R & 18 & -54 & -18 & 6.33 & $<0.001$ & 440 \\
\hline Cerebellum (VIII) & $\mathrm{R}$ & 16 & -62 & -44 & 5.84 & $<0.001$ & 42 \\
\hline Hippocampus & L & -30 & -38 & 2 & 5.68 & $<0.001$ & 71 \\
\hline Thalamus, striatum & $\mathrm{R}$ & 28 & -32 & 6 & 4.99 & 0.011 & 29 \\
\hline Thalamus & L & -16 & -26 & 6 & 5.65 & $<0.001$ & 77 \\
\hline
\end{tabular}

Local maxima of brain activations resulting from the contrast "nogo cue - go target" $>$ "go cue - nogo target" in MNI $x$-, $y$-, and $z$-coordinates with associated $Z$-score $\left(p_{F W E}<0.05, \mathrm{k}>5\right)$ and cluster extent in number of voxel (k). $R$, right; $L$, left.

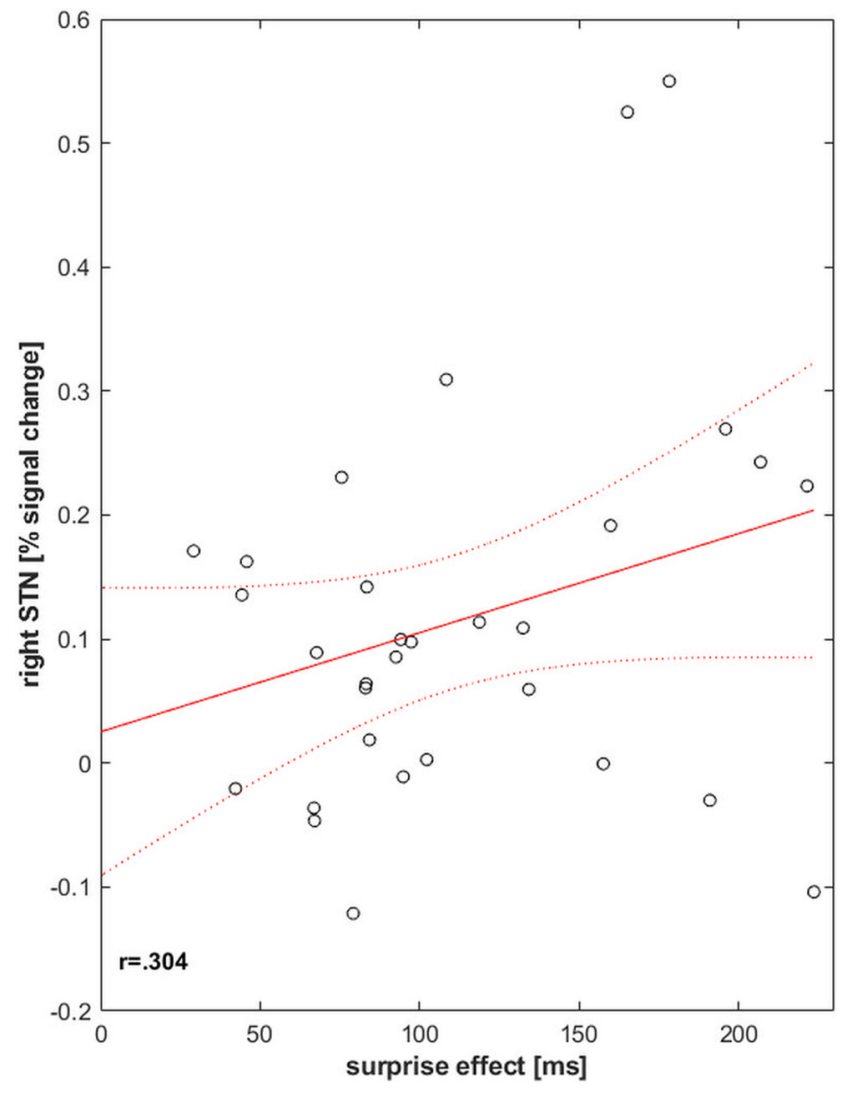

Figure 5. Surprise effect (i.e., the RT difference between unexpected and expected go trials) correlated significantly with percent signal change extracted from right STN region in unexpected go trials. This suggests that the more the STN region was recruited, the stronger the behavior was affected which is reflected in response slowing.

addition to the fronto-basal-ganglia system, unexpected inhibition and unexpected action recruited the IFJ and a parietal region within and around the intraparietal sulcus (IPS; Fig. 2, conjunction). Although both regions are commonly reported in neuroimaging studies on response inhibition, their function has specifically been linked to attentional processes (Chikazoe et al., 2009; Boehler et al., 2011; Cai and Leung, 2011; Corbetta and Shulman, 2011; Sebastian et al., 2016, 2017). Activity in these regions in response inhibition tasks is plausible since detecting and attentionally processing the salient stop stimulus is necessary before implementing unexpected control. While detecting a mismatch between current stimulus-driven bottom-up and ongoing goal-driven top-down information, the IFJ and IPS might promote the rapid interruption of ongoing cognitions through serial and parallel information transfer to the frontobasal-ganglia system in response inhibition and surprise tasks alike. Similarly, Wessel and Aron (2017) propose that signal detection, inhibition and attentional reorienting are part of a tightly linked cascade of interacting neural processes while assuming independence of interruption and attentional-reorienting. Importantly, in fMRI studies, which serve as the basis for key assumptions of the theory of unexpected events, action errors and unexpected action outcomes are associated with IFJ and IPS activity as well (cf. Wessel and Aron, 2017; see their Fig. 2). These findings challenge the notion that the interrupt system is entirely independent from attentional processes. fMRI studies can hardly dissociate whether attentional networks are elicited by the detection of unexpected stimuli or rather by the attentional reorienting subsequent to interruption. Future neurophysiological-neuroimaging studies with high temporal resolution and effective connectivity analyses are needed to unravel the time sequences in the recruitment of these networks (Schaum et al., 2020). In sum, while unexpected events trigger inhibitory control via the fronto-basal-ganglia stopping network, this process is closely contingent on attentional processes, and temporal interdependencies remain to be elucidated.

Findings regarding STN involvement are limited by the field strength of $3 \mathrm{~T}$ MRI which may not unequivocally localize BOLD signal to the STN (de Hollander et al., 2015). We nevertheless included STN region in our analyses given its pivotal importance for the theory of unexpected events (Wessel and Aron, 2017) and its causal role in surprise-induced behavioral inhibition (Fife et al., 2017). To increase anatomic specificity the presently used STN mask was derived from ultra-high resolution $7 \mathrm{~T}$ MRI (Keuken et al., 2014). STN activity during unexpected action did not survive Bonferroni correction. Bayesian statistics, however, provide moderate evidence that the present results are five times more likely under the null hypothesis (i.e., that unexpected action and unexpected inhibition equally recruit STN region) than under the alternative hypothesis. Moreover, correlation of STN activity and response slowing demonstrates behavioral relevance. One must acknowledge, however, that brain behavior correlations in small samples may be sensitive to outliers (Cremers et al., 2017). We thus provide preliminary support 
for the notion that unexpected events recruit the response inhibition network which interrupts ongoing behavior and cognition via the hyperdirect fronto-STN pathway (Nambu et al., 2002; Chen et al., 2020). These findings need to be replicated by studies using larger sample sizes and more suitable methods such as $7 \mathrm{~T}$ fMRI.

In sum, we provide evidence that unexpected action and unexpected inhibition following unexpected events recruit the same front-basal-ganglia network as response inhibition. The stronger recruitment was related to stronger response slowing. The present results support the theory of unexpected events and cannot be attributed to task or novelty effects.

\section{References}

Aron AR (2011) From reactive to proactive and selective control: developing a richer model for stopping inappropriate responses. Biol Psychiatry 69: e55-e68.

Aron AR, Poldrack RA (2006) Cortical and subcortical contributions to Stop signal response inhibition: role of the subthalamic nucleus. J Neurosci 26:2424-2433.

Aron AR, Robbins TW, Poldrack RA (2014) Inhibition and the right inferior frontal cortex: one decade on. Trends Cogn Sci 18:177-185.

Ballanger B, van Eimeren T, Moro E, Lozano AM, Hamani C, Boulinguez P, Pellecchia G, Houle S, Poon YY, Lang AE, Strafella AP (2009) Stimulation of the subthalamic nucleus and impulsivity: release your horses. Ann Neurol 66:817-824.

Bari A, Robbins TW (2013) Inhibition and impulsivity: behavioral and neural basis of response control. Prog Neurobiol 108:44-79.

Barto A, Mirolli M, Baldassarre G (2013) Novelty or surprise? Front Psychol 4:907.

Boehler CN, Appelbaum LG, Krebs RM, Chen L-C, Woldorff MG (2011) The role of stimulus salience and attentional capture across the neural hierarchy in a stop-signal task. PLoS One 6:e26386.

Boekel W, Forstmann BU, Keuken MC (2017) A test-retest reliability analysis of diffusion measures of white matter tracts relevant for cognitive control. Psychophysiology 54:24-33.

Cai W, Leung HC (2011) Rule-guided executive control of response inhibition: functional topography of the inferior frontal cortex. PLoS One 6: e20840.

Cai W, Ryali S, Chen T, Li CS, Menon V (2014) Dissociable roles of right inferior frontal cortex and anterior insula in inhibitory control: evidence from intrinsic and task-related functional parcellation, connectivity, and response profile analyses across multiple datasets. J Neurosci 34:1465214667.

Chen W, Hemptinne C. d, Miller AM, Leibbrand M, Little SJ, Lim DA, Larson PS, Starr PA (2020) Prefrontal-subthalamic hyperdirect pathway modulates movement inhibition in humans. Neuron 106:579-588.e3.

Chikazoe J, Jimura K, Asari T, Yamashita K-i, Morimoto H, Hirose S, Miyashita Y, Konishi S (2009) Functional dissociation in right inferior frontal cortex during performance of go/no-go task. Cereb Cortex 19:146-152.

Chumbley JR, Burke CJ, Stephan KE, Friston KJ, Tobler PN, Fehr E (2014) Surprise beyond prediction error. Hum Brain Mapp 35:4805-4814.

Cieslik EC, Mueller VI, Eickhoff CR, Langner R, Eickhoff SB (2015) Three key regions for supervisory attentional control: evidence from neuroimaging meta-analyses. Neurosci Biobehav Rev 48:22-34.

Collins AGE, Frank MJ (2016) Surprise! Dopamine signals mix action, value and error. Nat Neurosci 19:3-5.

Corbetta M, Shulman GL (2002) Control of goal-directed and stimulusdriven attention in the brain. Nat Rev Neurosci 3:201-215.

Corbetta M, Shulman GL (2011) Spatial neglect and attention networks. Annu Rev Neurosci 34:569-599.

Cremers HR, Wager TD, Yarkoni T (2017) The relation between statistical power and inference in fMRI. PLoS One 12:e184923.

de Hollander G, Keuken MC, Forstmann BU (2015) The subcortical cocktail problem; mixed signals from the subthalamic nucleus and substantia nigra. PLoS One 10:e0120572.

den Ouden HEM, Kok P, de Lange FG (2012) How prediction errors shape perception, attention, and motivation. Front Psychol 3:548.
Desikan RS, Ségonne F, Fischl B, Quinn BT, Dickerson BC, Blacker D, Buckner RL, Dale AM, Maguire RP, Hyman BT, Albert MS, Killiany RJ (2006) An automated labeling system for subdividing the human cerebral cortex on MRI scans into gyral based regions of interest. Neuroimage 31:968-980.

Donders FC (1969) On the speed of mental processes. Acta Psychol (Amst) 30:412-431.

Duann JR, Ide JS, Luo X, Li CS (2009) Functional connectivity delineates distinct roles of the inferior frontal cortex and presupplementary motor area in stop signal inhibition. J Neurosci 29:10171-10179.

Dutra IC, Waller DA, Wessel JR (2018) Perceptual surprise improves action stopping by nonselectively suppressing motor activity via a neural mechanism for motor inhibition. J Neurosci 38:1482-1492.

Eickhoff SB, Stephan KE, Mohlberg H, Grefkes C, Fink GR, Amunts K, Zilles K (2005) A new SPM toolbox for combining probabilistic cytoarchitectonic maps and functional imaging data. Neuroimage 25:1325-1335.

Eickhoff SB, Heim S, Zilles K, Amunts K (2006) Testing anatomically specified hypotheses in functional imaging using cytoarchitectonic maps. Neuroimage 32:570-582.

Eickhoff SB, Paus T, Caspers S, Grosbras M-H, Evans AC, Zilles K, Amunts K (2007) Assignment of functional activations to probabilistic cytoarchitectonic areas revisited. Neuroimage 36:511-521.

Ferdinand NK, Opitz B (2014) Different aspects of performance feedback engage different brain areas: disentangling valence and expectancy in feedback processing. Sci Rep 4:5986.

Fife KH, Gutierrez-Reed NA, Zell V, Bailly J, Lewis CM, Aron AR, Hnasko TS (2017) Causal role for the subthalamic nucleus in interrupting behavior. Elife 6:e27689.

Fouragnan E, Queirazza F, Retzler C, Mullinger KJ, Philiastides MG (2017) Spatiotemporal neural characterization of prediction error valence and surprise during reward learning in humans. Sci Rep 7:4762.

Fouragnan E, Retzler C, Philiastides MG (2018) Separate neural representations of prediction error valence and surprise: evidence from an fMRI meta-analysis. Hum Brain Mapp 39:2887-2906.

Gallistel CR (2009) The importance of proving the null. Psychol Rev 116:439-453.

Geiger LS, Moessnang C, Schäfer A, Zang Z, Zangl M, Cao H, van Raalten TR, Meyer-Lindenberg A, Tost H (2018) Novelty modulates human striatal activation and prefrontal-striatal effective connectivity during working memory encoding. Brain Struct Funct 223:3121-3132.

Georgiev D, Dirnberger G, Wilkinson L, Limousin P, Jahanshahi M (2016) In Parkinson's disease on a probabilistic Go/NoGo task deep brain stimulation of the subthalamic nucleus only interferes with withholding of the most prepotent responses. Exp Brain Res 234:1133-1143.

Gläscher J (2009) Visualization of group inference data in functional neuroimaging. Neuroinformatics 7:73-82.

Ide JS, Shenoy P, Yu AJ, Li C-SR (2013) Bayesian prediction and evaluation in the anterior cingulate cortex. J Neurosci 33:2039-2047.

Jahanshahi M, Obeso I, Rothwell JC, Obeso JA (2015) A fronto-striato-subthalamic-pallidal network for goal-directed and habitual inhibition. Nat Rev Neurosci 16:719-732.

JASP Team (2019). JASP (Version 0.11.1.0)[Computer software].

Johansen-Berg H, Behrens TEJ, Robson MD, Drobnjak I, Rushworth MFS, Brady JM, Smith SM, Higham DJ, Matthews PM (2004) Changes in connectivity profiles define functionally distinct regions in human medial frontal cortex. Proc Natl Acad Sci USA 101:13335-13340.

Keuken MC, Bazin P-L, Crown L, Hootsmans J, Laufer A, Müller-Axt C, Sier R, van der Putten EJ, Schäfer A, Turner R, Forstmann BU (2014) Quantifying inter-individual anatomical variability in the subcortex using 7 T structural MRI. Neuroimage 94:40-46.

Lanska DJ (2009) The history of movement disorders. In: History of neurology, pp 501-546. San Diego: Elsevier.

Levy BJ, Wagner AD (2011) Cognitive control and right ventrolateral prefrontal cortex: reflexive reorienting, motor inhibition, and action updating. Ann NY Acad Sci 1224:40-62.

Logan GD, Cowan WB (1984) On the ability to inhibit thought and action: a theory of an act of control. Psychol Rev 91:295-327.

Marmor O, Rappel P, Valsky D, Bick AS, Arkadir D, Linetsky E, Peled O, Tamir I, Bergman H, Israel Z, Eitan R (2020) Movement context modulates neuronal activity in motor and limbic-associative domains of the human parkinsonian subthalamic nucleus. Neurobiol Dis 136:104716. 
Marsden CD, Obeso JA, Zarranz JJ, Lang AE (1985) The anatomical basis of symptomatic hemidystonia. Brain 108:463-483.

Nambu A, Tokuno H, Takada M (2002) Functional significance of the cortico-subthalamo-pallidal 'hyperdirect' pathway. Neurosci Res 43:111117.

Nichols T, Brett M, Andersson J, Wager T, Poline JB (2005) Valid conjunction inference with the minimum statistic. Neuroimage 25:653-660.

Niv Y, Daniel R, Geana A, Gershman SJ, Leong YC, Radulescu A, Wilson RC (2015) Reinforcement learning in multidimensional environments relies on attention mechanisms. J Neurosci 35:8145-8157.

Oldfield RC (1971) The assessment and analysis of handedness: the Edinburgh inventory. Neuropsychologia 9:97-113.

Ranganath C, Rainer G (2003) Neural mechanisms for detecting and remembering novel events. Nat Rev Neurosci 4:193-202.

Schachar R, Logan GD, Robaey P, Chen S, Ickowicz A, Barr C (2007) Restraint and cancellation: multiple inhibition deficits in attention deficit hyperactivity disorder. J Abnorm Child Psychol 35:229-238.

Schaum M, Pinzuti E, Sebastian A, Lieb K, Fries P, Mobascher A, Jung P, Wibral M, Tüscher O (2020) Cortical network mechanisms of response inhibition. biorxiv. doi: 10.1101/2020.02.09.940841.

Sebastian A, Pohl MF, Klöppel S, Feige B, Lange T, Stahl C, Voss A, Klauer KC, Lieb K, Tüscher O (2013) Disentangling common and specific neural subprocesses of response inhibition. Neuroimage 64:601-615.

Sebastian A, Jung P, Neuhoff J, Wibral M, Fox PT, Lieb K, Fries P, Eickhoff SB, Tüscher O, Mobascher A (2016) Dissociable attentional and inhibitory networks of dorsal and ventral areas of the right inferior frontal cortex: a combined task-specific and coordinate-based meta-analytic fMRI study. Brain Struct Funct 221:1635-1651.
Sebastian A, Rössler K, Wibral M, Mobascher A, Lieb K, Jung P, Tüscher O (2017) Neural Architecture of selective stopping strategies: distinct brain activity patterns are associated with attentional capture but not with outright stopping. J Neurosci 37:9785-9794.

Swick D, Ashley V, Turken U (2011) Are the neural correlates of stopping and not going identical? Quantitative meta-analysis of two response inhibition tasks. Neuroimage 56:1655-1665.

Wagenmakers EJ, Morey RD, Lee MD (2016) Bayesian benefits for the pragmatic researcher. Curr Dir Psychol Sci 25:169-176.

Wagner J, Wessel JR, Ghahremani A, Aron AR (2018) Establishing a right frontal beta signature for stopping action in scalp EEG: implications for testing inhibitory control in other task contexts. J Cogn Neurosci 30:107118.

Wessel JR (2018) A neural mechanism for surprise-related interruptions of visuospatial working memory. Cereb Cortex 28:199-212.

Wessel JR, Aron AR (2013) Unexpected events induce motor slowing via a brain mechanism for action-stopping with global suppressive effects. J Neurosci 33:18481-18491.

Wessel JR, Aron AR (2017) On the globality of motor suppression: unexpected events and their influence on behavior and cognition. Neuron 93:259-280.

Wessel JR, Huber DE (2019) Frontal cortex tracks surprise separately for different sensory modalities but engages a common inhibitory control mechanism. PLoS Comput Biol 15:e1006927.

Wessel JR, Jenkinson N, Brittain J-S, Voets SHEM, Aziz TZ, Aron AR (2016) Surprise disrupts cognition via a fronto-basal ganglia suppressive mechanism. Nat Commun 7:11195. 